\section{Adaptação transcultural da versão brasileira da Escala do Impacto do Evento - Revisada (IES-R)}

\author{
Cross-cultural adaptation of the Brazilian version of \\ the Impact of Events Scale-Revised (IES-R)
}

\begin{abstract}
The Impact of Events Scale-Revised (IES-R) is used to screen for post-traumatic stress disorder (PTSD). The aim of this study was to assess the cross-cultural adaptation of the IES-R. The scale was translated into Brazilian Portuguese and culturally adapted. Reliability and validity were evaluated in 45 individuals divided into three groups of 15 (without PTSD, with PTSD, and treated for PTSD). Reliability was evaluated using intraclass correlation coefficient - ICC and Cronbach's alfa. Discriminant validity was evaluated by comparing mean IES-R scores in the three groups. The area under the ROC curve (AUC) was evaluated to determine cut-offs with higher sensitivity (s) and specificity (e) using the clinical interview (DSM-IV) as reference. The IES-R showed good reliability (ICC $=1$; alfa from 0.75 to 0.93 ). The mean IES-R scores $(p<0.05)$ and ROC curve had good discriminant validity for a cut-off of 5.6 $(s=0.80, e=0.70$ and $A U C=0.81)$. The Brazilian version of IES-R showed good properties and can be a useful screening tool for PTSD.
\end{abstract}

Post-Traumatic Stress Disorders; Diagnosis; Validation Studies; Translating; Questionnaires
Andrea Vannini Santesso Caiuby 1

Shirley Silva Lacerda 1

Maria Inês Quintana 1

Thais Suemi Torii 1

Sergio Baxter Andreoli 1

\section{Introdução}

O transtorno do estresse pós-traumático é definido como um conjunto de sintomas associados a um evento traumático ${ }^{1} \mathrm{~A}$ Escala do Impacto do Evento-Revisada (IES-R) tem sido referida como o instrumento de rastreamento da sintomatologia do transtorno do estresse pós-traumático que demonstra melhor validade discriminante e utilidade diagnóstica 2 , podendo ser utilizada em qualquer fase do desenvolvimento dos sintomas (agudo, crônico e tardio). Estudos com a IES-R demonstraram bons escores de consistência interna, com coeficientes de Alfa de Cronbach de 0,81 a $0,963,4,5,6,7,8$, de validade com $100 \%$ de sensibilidade e $78 \%$ de especificidade 9 .

A IES-R é o instrumento de rastreio mais citado na literatura, mas nenhum estudo foi feito no Brasil e poucos foram os que realizaram a validação da IES-R contra entrevistas clínicas estruturadas. Assim, o presente trabalho teve como objetivos o desenvolvimento de uma versão da IES-R adaptada para o português do Brasil, avaliação da confiabilidade e validade discriminante. 


\section{Método}

\section{Instrumento}

A IES-R é uma escala do tipo likert no qual o indivíduo responde às questões baseando-se nos 7 dias anteriores à aplicação da escala e desenvolvida para autoaplicação. A escala é composta de 22 itens distribuídos em 3 subescalas (evitação, intrusão e hiperestimulação) que contemplam os critérios de avaliação de transtorno do estresse pós-traumático publicados no DSM-IV 1 . O escore para cada questão varia de 0 a 4 pontos e o cálculo do escore de cada subescala é obtido por meio da média dos itens que compõem as subescalas evitação, intrusão e hiperestimulação, desconsiderando as questões não respondidas. O escore total é a soma dos escores das subescalas $3,4,10$.

\section{Participantes}

Participaram deste estudo 45 pacientes maiores de 18 anos que sofreram eventos traumáticos de violência urbana e doméstica, provenientes de 2 hospitais públicos da cidade de São Paulo, Brasil. Os dados foram coletados de outubro de 2007 a abril de 2008 e os participantes foram divididos em 3 grupos de 15: grupo sem transtorno do estresse pós-traumático (pacientes que não desenvolveram transtorno); grupo com transtorno do estresse pós-traumático (com sintomas de transtorno); e grupo tratado (sem sintomas de transtorno após o tratamento). O diagnóstico de transtorno do estresse pós-traumático foi realizado por médico psiquiatra, baseado nos critérios diagnósticos do DSM-IV 1, e a participação dos pacientes foi condicionada ao aceite do Termo de Consentimento Livre e Esclarecido (aprovado pelo Comitê de Ética em Pesquisa do Hospital Municipal do Campo Limpo e da Universidade Federal de São Paulo). Os sujeitos que apresentavam sintomas psicóticos e déficit cognitivo grave foram excluídos.

\section{Procedimentos}

A adaptação transcultural foi realizada conforme as diretrizes sugeridas por Beanton et al. 11 e, assim, a tradução para o português do Brasil foi realizada por 2 tradutores nativos em português e tendo o inglês como segunda língua, que realizaram as traduções de forma independente. Os dois tradutores elaboraram uma versão consensual em português que foi traduzida de volta para o inglês por um terceiro tradutor bilíngue, e então comparada com a versão original. Posteriormente, a versão em português da IES-R foi lida por 10 sujeitos, maiores de 18 anos, para avaliação da compreensão e aceitabilidade.

A avaliação da confiabilidade foi realizada usando-se o cálculo do Coeficiente de Correlação Intraclasse (ICC), e da consistência interna medida pelo Alfa de Cronbach. Quatro psicólogos foram treinados para aplicação, sendo divididos em 2 pares compostos por um avaliador e um observador. O avaliador foi o responsável pela leitura das questões enquanto ambos realizavam as anotações das respostas de forma simultânea e independente. Tanto o aplicador quanto o observador eram cegos em relação ao grupo, e as aplicações da escala foram realizadas em condições similares para os 3 grupos.

A avaliação dos escores de validade discriminante foi realizada por meio da comparação entre as médias dos escores totais entre os 3 grupos e pela análise da curva ROC para determinação das medidas de sensibilidade e especificidade. O diagnóstico clínico de transtorno do estresse pós-traumático foi usado como padrão ouro na análise da curva ROC, e o ponto de corte foi estabelecido com a finalidade de contemplar as melhores sensibilidade e especificidade.

\section{Análise dos dados}

Os dados foram analisados usando-se o Statistic Package for Social Science 16.0 (SPSS Inc., Chicago, Estados Unidos). Foram usados procedimentos de estatística descritiva (frequência, médias e desvios-padrão) para os dados demográficos; Coeficiente de Correlação Intraclasse (ICC) e Alfa de Cronbach para obtenção de escore de confiabilidade; e teste de ANOVA One-Way com teste de Post Hoc de Bonferroni e análise da curva ROC para o estudo de validade discriminante.

\section{Resultados}

A comparação da versão original da IES-R com a retrotradução da versão consensual em português não apresentou diferenças que justificassem alterações na versão em português. Contudo, após a aplicação em 10 participantes, constataram-se dificuldades na compreensão da instrução e dos itens 5, 7, 9, 10 e 12, sendo necessário realizar adaptações (Figura 1). Após as adaptações, chegou-se à versão final da IES-R em português (Figura 2).

O tempo de aplicação da versão em português da IES-R foi de aproximadamente $15 \mathrm{mi}$ nutos. Os três grupos diferenciaram quanto ao gênero predominante, anos de estudo e tipo de evento estressor (Tabela 1). Para a análise da curva ROC o grupo de pacientes sem transtorno do 
Principais mudanças durante a fase de tradução e retrotradução.

\begin{tabular}{|c|c|c|}
\hline Itens do original em inglês & Tradução consensual do item em português & Versão final do item em português \\
\hline $\begin{array}{l}\text { Instructions } \\
\text { "Below is a list of difficulties people sometimes have } \\
\text { after stressful life events. Please read each item, and } \\
\text { then indicate how distressing each difficulty has been } \\
\text { for you DURING THE PAST SEVEN DAYS with respect } \\
\text { to , how much } \\
\text { were you distressed or bothered by these difficulties?" }\end{array}$ & $\begin{array}{l}\text { Instrução } \\
\text { "Listamos abaixo algumas dificuldades que as pessoas } \\
\text { algumas vezes apresentam após passar por alguns } \\
\text { eventos de vida estressantes. Por favor, leia cada item e } \\
\text { depois indique o quanto cada situação foi estressante } \\
\text { para você DURANTE OS ÚLTIMOS SETE DIAS após } \\
\text { ter ocorrido, o quanto } \\
\text { você ficou estressado ou se incomodou com essas } \\
\text { dificuldades?" }\end{array}$ & $\begin{array}{l}\text { Instrução } \\
\text { "Listamos abaixo dificuldades que as pessoas } \\
\text { algumas vezes apresentam, após passar por eventos } \\
\text { estressantes. Com relação às memórias do evento } \\
\text { estressor por favor, leia cada } \\
\text { item abaixo e depois marque com um X a coluna } \\
\text { que melhor corresponde a seu nível de estresse, nos } \\
\text { últimos } 7 \text { dias". }\end{array}$ \\
\hline $\begin{array}{l}\text { Item } \mathbf{5} \\
\text { "I avoided letting myself get upset when I thought } \\
\text { about it or was reminded of it" }\end{array}$ & $\begin{array}{l}\text { Item } \mathbf{5} \\
\text { "Eu evitava me deixar ficar chateado quando pensava } \\
\text { sobre a situação ou era lembrado dela" }\end{array}$ & $\begin{array}{l}\text { Item } \mathbf{5} \\
\text { "Eu evitava ficar chateado quando pensava na situação } \\
\text { ou era lembrado dela" }\end{array}$ \\
\hline $\begin{array}{c}\text { Item } 7 \\
\text { "I felt as if it hadn't happened or wasn't real" }\end{array}$ & $\begin{array}{l}\text { Item } 7 \\
\text { "Eu senti como se não tivesse acontecido ou como se } \\
\text { não fosse real" }\end{array}$ & $\begin{array}{l}\text { Item } 7 \\
\text { "Eu senti como se não tivesse passado pela situação } \\
\text { ou como se não fosse real" }\end{array}$ \\
\hline $\begin{array}{c}\text { Item } 9 \\
\text { "Pictures about it popped into my mind" }\end{array}$ & $\begin{array}{l}\text { Item } 9 \\
\text { "Imagens sobre a situação estalavam dentro da minha } \\
\text { mente" }\end{array}$ & $\begin{array}{l}\text { Item } 9 \\
\text { "Imagens sobre a situação saltavam em minha } \\
\text { mente" }\end{array}$ \\
\hline $\begin{array}{l}\text { Item } 10 \\
\text { "I was jumpy and easily startled" }\end{array}$ & $\begin{array}{l}\text { Item } 10 \\
\text { "Eu ficava sobressaltado e facilmente em estado de } \\
\text { alerta" }\end{array}$ & $\begin{array}{c}\text { Item } 10 \\
\text { "Eu ficava sobressaltado e facilmente alarmado" }\end{array}$ \\
\hline $\begin{array}{l}\text { Item } 12 \\
\text { "I was aware that I still had a lot of feelings about it, but } \\
\text { I didn't deal with them" }\end{array}$ & $\begin{array}{l}\text { Item } 12 \\
\text { "Eu estava ciente de que ainda tinha muitas emoções } \\
\text { ligadas à situação, mas eu não tratei delas" }\end{array}$ & $\begin{array}{l}\text { Item } 12 \\
\text { "Eu sabia que ainda tinha muitas emoções ligadas à } \\
\text { situação, mas as evitei" }\end{array}$ \\
\hline
\end{tabular}

estresse pós-traumático e o de pacientes tratados formaram um único grupo e não foi mais encontrada diferença significante entre este grupo e o grupo de pacientes com transtorno do estresse pós-traumático.

Os escores do ICC demonstraram total concordância ( $\mathrm{ICC}=1,000$ ) entre o aplicador e o observador para todas as subescalas da IES-R. A avaliação da consistência interna apresentou coeficientes de Alfa de Cronbach de 0,933 para o escore total, 0,754 para a subescala evitação, 0,882 para a intrusão e de 0,878 para a subescala hiperestimulação.

Quanto aos escores da IES-R, a análise de variância (ANOVA) encontrou diferença significante entre as médias dos três grupos $(\mathrm{F}[2]=8,867$; $\mathrm{p}=0,001$ ) (Tabela 3 ). Análises post hoc demonstraram que o grupo de pacientes com transtorno do estresse pós-traumático obteve pontuação total estatisticamente mais alta do que os grupos de pacientes sem transtorno do estresse póstraumático $(\mathrm{p}=0,001)$ e pacientes tratados $(\mathrm{p}=$ 0,008). A análise da curva Roc apresentou a área sob a curva de 0,814 , com sensibilidade de 0,80 e especificidade de 0,70 para um ponto de corte de 5,6 pontos.

\section{Discussão}

O processo de adaptação transcultural teve por objetivo encontrar equivalência entre a forma original da escala e a versão em português e, ao final, a não ocorrência de dificuldades de compreensão demonstrou que o trabalho foi realizado de forma adequada para aplicação da escala ${ }^{11}$. 
Figura 2

Versão da Escala do Impacto do Evento - Revisada (IES-R) traduzida para a língua portuguesa.

Listamos abaixo as dificuldades que as pessoas algumas vezes apresentam, após passar por eventos estressantes. Com relação às memórias do evento estressor por favor, leia cada item abaixo e depois marque com um $\mathrm{X}$ a coluna que melhor corresponde a seu nível de estresse, nos últimos 7 dias.

\begin{tabular}{|c|c|c|c|c|c|c|}
\hline & & Nem um pouco & Um pouco & Moderadamente & Muito & Extremamente \\
\hline 1. & $\begin{array}{l}\text { Qualquer lembrança trazia de volta } \\
\text { sentimentos sobre a situação }\end{array}$ & 0 & 1 & 2 & 3 & 4 \\
\hline 2. & Eu tinha problemas em manter o sono & 0 & 1 & 2 & 3 & 4 \\
\hline 3. & $\begin{array}{l}\text { Outros acontecimentos faziam com que } \\
\text { eu ficasse pensando sobre a situação }\end{array}$ & 0 & 1 & 2 & 3 & 4 \\
\hline 4. & Eu me sentia irritável e bravo & 0 & 1 & 2 & 3 & 4 \\
\hline 5. & $\begin{array}{l}\text { Eu evitava ficar chateado quando } \\
\text { pensava sobre a situação ou era } \\
\text { lembrado dela }\end{array}$ & 0 & 1 & 2 & 3 & 4 \\
\hline 6. & $\begin{array}{l}\text { Eu pensava sobre a situação mesmo } \\
\text { quando não tinha intenção de pensar }\end{array}$ & 0 & 1 & 2 & 3 & 4 \\
\hline 7. & $\begin{array}{l}\text { Eu sentia como se não tivesse passado } \\
\text { pela situação ou como se não fosse real }\end{array}$ & 0 & 1 & 2 & 3 & 4 \\
\hline 8. & $\begin{array}{l}\text { Eu me mantive longe de coisas que } \\
\text { pudessem relembrar a situação }\end{array}$ & 0 & 1 & 2 & 3 & 4 \\
\hline 9. & $\begin{array}{l}\text { Imagens sobre a situação saltavam em } \\
\text { minha mente }\end{array}$ & 0 & 1 & 2 & 3 & 4 \\
\hline 10. & $\begin{array}{l}\text { Eu ficava sobressaltado e facilmente } \\
\text { alarmado }\end{array}$ & 0 & 1 & 2 & 3 & 4 \\
\hline 11. & Eu tentei não pensar sobre a situação & 0 & 1 & 2 & 3 & 4 \\
\hline & $\begin{array}{l}\text { Eu sabia que ainda tinha muitas emoções } \\
\text { ligadas à situação, mas as evitei }\end{array}$ & 0 & 1 & 2 & 3 & 4 \\
\hline 13. & $\begin{array}{l}\text { Meus sentimentos sobre a situação } \\
\text { estavam como que entorpecidos }\end{array}$ & 0 & 1 & 2 & 3 & 4 \\
\hline 14. & $\begin{array}{l}\text { Eu me peguei agindo ou sentindo como } \\
\text { se estivesse de volta à situação }\end{array}$ & 0 & 1 & 2 & 3 & 4 \\
\hline 15. & Eu tive problemas para dormir & 0 & 1 & 2 & 3 & 4 \\
\hline 16. & $\begin{array}{l}\text { Eu tive ondas de fortes emoções relativas } \\
\text { à situação }\end{array}$ & 0 & 1 & 2 & 3 & 4 \\
\hline & $\begin{array}{l}\text { Eu tentei retirar a situação da minha } \\
\text { memória }\end{array}$ & 0 & 1 & 2 & 3 & 4 \\
\hline 18. & Eu tive problemas de concentração & 0 & 1 & 2 & 3 & 4 \\
\hline 19. & $\begin{array}{l}\text { Lembranças da situação faziam com que } \\
\text { eu tivesse reações físicas, como suores, } \\
\text { problemas para respirar, náuseas ou } \\
\text { coração disparado }\end{array}$ & 0 & 1 & 2 & 3 & 4 \\
\hline & Eu tive sonhos sobre a situação & 0 & 1 & 2 & 3 & 4 \\
\hline 21. & Eu me sentia atento ou na defensiva & 0 & 1 & 2 & 3 & 4 \\
\hline 22. & Eu tentei não falar sobre a situação & 0 & 1 & 2 & 3 & 4 \\
\hline
\end{tabular}


Dados demográficos e escores da Escala do Impacto do Evento - Revisada (IES-R) dos 3 grupos avaliados.

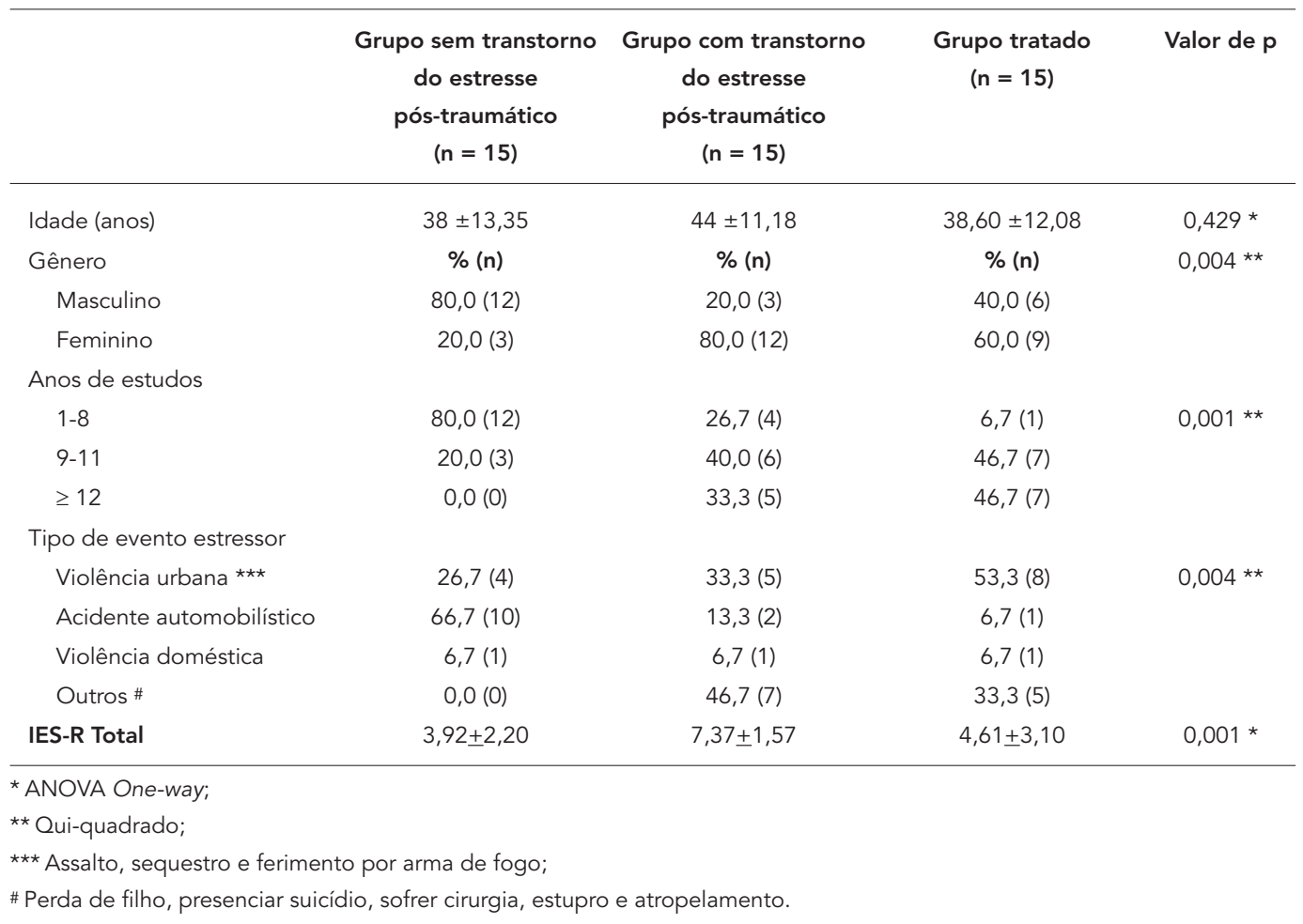

A avaliação da confiabilidade da IES-R medida pelo ICC exibiu total concordância entre o aplicador e o observador, apresentando bons índices de confiabilidade, assim como os obtidos em outros estudos $3,4,5,6,7,8,9$.

O estudo da validade da versão brasileira da IES-R demonstrou que a escala foi capaz de discriminar estatisticamente o grupo com transtorno do estresse pós-traumático dos grupos sem transtorno. Porém, os 3 grupos estudados mostraram-se heterogêneos em relação a gênero, anos de estudo e eventos estressores, sendo que a diferença em anos de estudo poderia explicar parte da diferença encontrada nos escores da escala 12. Contudo, quando os pacientes sem transtorno do estresse pós-traumático e pacientes tratados foram agrupados em um único grupo, as diferenças demográficas com o grupo com transtorno do estresse pós-traumático passaram a não mais existir.

A análise da curva ROC demonstrou que a IES-R conseguiu discriminar com bons índices de sensibilidade e especificidade os pacientes com transtorno do estresse pós-traumático de pacientes sem transtorno do estresse pós-traumático, sendo selecionado o ponto de corte de
5,6. A diferença encontrada entre o ponto de corte aqui sugerido e o de outros estudos pode ser explicada por 3 vertentes: alguns estudos optam pela simples soma dos itens, desconsiderando o cálculo da média dos itens conforme indicado pelos autores $3,4,5,6,7$; os estudos de validação foram realizados com sujeitos que sofreram diferentes eventos estressores e avaliados em diferentes tempos após a ocorrência 4,5,8; o ponto de corte selecionado no presente trabalho privilegiou os melhores índices de sensibilidade e especificidade para o rastreio da sintomatologia de transtorno do estresse pós-traumático, diferentemente de alguns autores que priorizaram outras necessidades clínicas 4,7 .

Assim, a versão da IES-R adaptada para o português do Brasil demonstrou ser de fácil aplicação e compreensão, bem como exibiu bons índices de confiabilidade e validade, sendo a sua aplicação indicada no rastreio de sintomatologia de transtorno do estresse pós-traumático. Esses resultados sustentam a indicação de uso na população brasileira, mas outros estudos que avaliem diferentes características sociodemográficas e que utilizem outras medidas de validade podem complementar os resultados apresentados. 


\section{Resumo}

$A$ Escala de Impacto do Evento - Revisada (IES-R) é utilizada no rastreio de transtorno de estresse póstraumático. O objetivo do estudo foi avaliar a adaptação transcultural da IES-R. Realizou-se a tradução e adaptação da escala para o português do Brasil. Confiabilidade e validade foram avaliadas entre $45 \mathrm{su}$ jeitos distribuídos em 3 grupos de 15: sem transtorno, com transtorno e tratados para transtorno. A confiabilidade foi avaliada pelo Coeficiente de Correlação Intraclasse (ICC) e pelo alfa de Cronbach ( $\alpha$ ). A validade discriminante foi calculada pela comparação dos escores médios de IES-R nos 3 grupos. A área sobre a curva ROC (AUC) foi avaliada para determinar os pontos de corte com maiores sensibilidade (s) e especificidade (e), utilizando-se a entrevista clínica (DSM-IV) como referência. A IES-R exibiu boa confiabilidade (ICC $=1$; $\alpha$ entre 0,75 e 0,93). Os escores médios da IES-R $(p<0,05)$ e a curva ROC mostraram boa validade discriminante para o ponto de corte de 5,6 $(s=0,80, e=0,70$ e AUC $=$ 0,81 ). A versão brasileira da IES-R apresentou boas propriedades e mostrou-se eficiente no rastreio de transtorno de estresse pós-traumático.

Transtornos de Estresse Pós-Traumáticos; Diagnóstico; Estudos de Validação; Tradução (Processo); Questionários

\section{Colaboradores}

A. V. S. Caiuby foi responsável pelo trabalho de campo, análises do material e redação do texto do artigo. S. S. Lacerda foi responsável pela análise estatística, contribuições importantes para a discussão e revisão do conteúdo intelectual do artigo. M. I. Quintana trabalhou em conjunto com a primeira autora na tradução, retrotradução e adaptação do instrumento. T. S. Torii contribuiu na revisão do conteúdo intelectual e referências bibliográficas do artigo. S. B. Andreoli foi responsável pela revisão do conteúdo intelectual do artigo.

\section{Agradecimentos}

Projeto financiado pela Fundação de Amparo à Pesquisa do Estado de São Paulo (FAPESP), processo $\mathrm{n}^{\circ}$. 05/55513-6. A autora agradece a concessão de bolsa de doutorado da Coordenação de Aperfeiçoamento de Pessoal de Nível Superior e à equipe do Núcleo de Estatística e Metodologia Aplicada (NEMAP) do Centro de Estudos do Departamento de Psiquiatria da Universidade Federal de São Paulo.

\section{Referências}

1. American Psychiatric Association. DSM-IV (TR): manual diagnóstico e estatístico de transtornos mentais. Porto Alegre: Artes Médicas; 2002.

2. Adkins JW, Weathers FW, McDevitt-Murphy M, Daniels JB. Psychometric properties of seven selfreport measures of posttraumatic stress disorder in college students with mixed civilian trauma exposure. J Anxiety Disord 2008; 22:1393-402.

3. Weiss DSMCR. Impact of Event Scale (IES-R). In: Wilson T, Keane T, editors. The Impact of evente Scale - Revised: assessing psychological trauma and PTSD. New York: Guilford Press; 1997. p. 399411.

4. Creamer M, Bell R, Failla S. Psychometric properties of the Impact of Event Scale - Revised. Behav Res Ther 2003; 41:1489-96.
5. Asukai N, Kato H, Kawamura N, Kim Y, Yamamoto $\mathrm{K}$, Kishimoto J, et al. Reliability and validity of the Japanese-language version of the impact of event scale-revised (IES-R-J): four studies of different traumatic events. J Nerv Ment Dis 2002; 190: 175-82.

6. Brunet A, St-Hilaire A, Jehel L, King S. Validation of a French version of the impact of event scalerevised. Can J Psychiatry 2003; 48:56-61.

7. Rash CJ, Coffey SF, Baschnagel JS, Drobes DJ, Saladin ME. Psychometric properties of the IES-R in traumatized substance dependent individuals with and without PTSD. Addict Behav 2008; 33:1039-47. 
8. Baumert J, Simon H, Gundel H, Schmitt C, Ladwig KH. The Impact of Event Scale - Revised: evaluation of the subscales and correlations to psychophysiological startle response patterns in survivors of a life-threatening cardiac event: an analysis of 129 patients with an implanted cardioverter defibrillator. J Affect Disord 2004; 82:29-41.

9. Sveen J, Low A, Dyster-Aas J, Ekselius L, Willebrand $\mathrm{M}$, Gerdin B. Validation of a Swedish version of the Impact of Event Scale-Revised (IES-R) in patients with burns. J Anxiety Disord 2010; 24:618-22.

10. Horowitz M, Wilner N, Alvarez W. Impact of Event Scale: a measure of subjective stress. Psychosom Med 1979; 41:209-18.
11. Beaton DE, Bombardier C, Guillemin F, Ferraz MB. Guidelines for the process of cross-cultural adaptation of self-report measures. Spine 2000; 25:3186-91.

12. van der Ploeg E, Mooren TT, Kleber RJ, van der Velden PG, Brom D. Construct validation of the Dutch version of the impact of event scale. Psychol Assess 2004; 16:16-26.

Recebido em 02/Dez/2010

Versão final reapresentada em 07/Nov/2011

Aprovado em 30/Nov/2011 